\title{
TXOP Combinatorial Problem in IEEE 802.11e HCCA Networks
}

\author{
Ming-Hua Cheng, Cheng-Han Lin, Wen-Shyang Hwang, and Feng-Cheng Yu
}

\begin{abstract}
The Hybrid Coordination Function (HCF) was proposed in IEEE 802.11e, including the competition-based Enhanced Distributed Channel Access (EDCA) and polling-based HCF Controlled Channel Access (HCCA). This paper mainly carries on the research in the HCCA mechanism. In the HCCA mechanism, the Sample Scheduler offers a way to determine the time duration of the Transmission Opportunity (TXOP). The determination of TXOP is calculated by the equation according to two parameters of transmission, including the Mean Data Rate and Packet Size. Next, Sample Scheduler distributes the result of the TXOP to the QSTA. However, QSTAs are not able to completely utilize the allocated TXOP, which causes the waste of remaining TXOPs. The remaining TXOPs of each QSTA are wasted. Therefore, this paper focuses on allocating the remaining TXOP to QSTAs that can transmits more data packets and reduces the packet dropped rate. The simulation results show that the proposed scheme increases the system throughput and decreases the packet loss rate by utilizing the remaining TXOP.
\end{abstract}

\section{Index Terms-IEEE 802.11e, QoS, HCCA, TXOP.}

\section{INTRODUCTION}

With the development of information technology, the technologies of wireless network gradually mature. It can be seen everywhere in the products correlated with wireless network, at home network or public places. However, according to the development of multimedia in recent years, the quality of traditional IEEE 802.11 [1] mechanism cannot support the QoS service. In order to enable the Wireless Local Area Network (WLAN) to offer steady service quality as Ethernet, the standard of IEEE 802.11e was qualified in 2005 [2]. In IEEE 802.11e, the HCF was proposed to support QoS service. There are two types of channel access in the HCF, including the EDCA and HCCA. In addition, the mechanism of TXOP in HCF transmission protocol has been defined to enable the QSTA to transmit a lot of data frames within the period of TXOP. The TXOP solves the problem that only one data frame can be transmitted at one time in the traditional IEEE 802.11 standard. In the IEEE 802.11e standard, the polling will be carried out after performing the Sample Schedule. The Sample Schedule initiates the Controlled Access Phase (CAP) in one regular cycle by its length. The length of this cycle is called the Service Interval (SI). According to the determination of TXOP, the QoS-Station

Manuscript received December 18, 2014; revised October 23, 2015.

Wen-Shyang Hwang, Wen-Shyang Hwang, and Feng-Cheng Yu are with the National Kaohsiung University of Applied Sciences, Kaohsiung, Taiwan (e-mail: wshwang@mail.ee.kuas.edu.tw).

Cheng-Han Lin is with the Program of Information Technology, Fooyin University, Taiwan.
(QSTA) can transmit a lot of data frames within this period of TXOP time. The remaining TXOP will become a waste of system resource [3]-[9]. Accordingly, G. Cecchetti proposed the W-CBS [3] to arrange the Maximum Service Interval (MSI) to each QSTA based on the weighted TXOP. However, if the calculated TXOP value is relatively small, the QSTA cannot transmit packet. If the calculated TXOP value is relatively high, there will be a lot of remaining TXOP. Therefore, this paper basically improves the computing technology of the TXOP and enables the QSTA to increase throughput. The structure of this paper is as follows. In section two, we introduce the calculation of IEEE 802.11e HCCA Sample Scheduler and the related works. In section three, we propose an Extra-TXOP (X-TXOP) mechanism. In section four, we introduce the proposed mechanism and conduct a comparison to analyze the mechanism. Finally, section five is the conclusion of this paper.

\section{BACKGROUND AND RELATED WORKS}

\section{A. IEEE 802.11e HCCA}

The mechanism of HCCA utilizes the central control point (Hybrid Coordinator, HC) to control the access of wireless media. When joining the flow, the QSTA will send out an ADDTS request for the HC. Then the HC will be on the TSPEC field of the ADDTS request. In the IEEE 802.11e standard, the Sample Schedule will start the Controlled Access Phase (CAP) in one regular cycle by its length. The length of this cycle is called the Service Interval (SI). The procedure begins from the calculation of the SI. The Beacon Interval (BI) should not be greater than the Maximum Service Interval (MSI) value of all Traffic Stream (TSs). The SI is calculated as (1):

$$
\mathrm{SI}=\left\lceil\frac{\mathrm{BI}}{\mathrm{BI} / \min _{0 \leq i \leq n}\left\{\mathrm{SI}_{i}\right\}}\right\rceil
$$

Then, the $N_{i}$ and $\mathrm{TXOP}_{i}$ are determined as Eq. (2) and (3) when the times of $\mathrm{TS}_{i}$ reaches $N_{i}$.

$$
N_{i}=\left\lceil\frac{\rho_{i} \times \mathrm{SI}}{L_{i}}\right\rceil
$$

$$
\mathrm{TXOP}_{i}=\max \left(\frac{N_{i} \times L_{i}}{R_{i}}+o, \frac{M}{R_{i}}+o\right)
$$

where $L_{i}$ is the nominal MAC Service Data Unit (MSDU) size 
of the $\mathrm{TS}_{i}, R_{i}$ is the minimum PHY rate of $\mathrm{TS}_{i}, M$ is the maximum MSDU size and $o$ is the overhead. The summation of all TXOP of each QSTA is enough to provide service for the new flow and for the HC to check the bandwidth. The calculation is as Eq. (4):

$$
\frac{\mathrm{TXOP}_{k+1}}{\mathrm{SI}}+\sum_{i=1}^{k} \frac{\mathrm{TXOP}_{i}}{\mathrm{SI}} \leq \frac{\mathrm{SI}-T_{C P}}{\mathrm{SI}}
$$

where $k$ is the current quantity of TS and $k+1$ represents the TS that is newly joined. The TCP is the time of cycle of the competition. Via the SI and TXOP calculated by the above equations.

\section{B. W-CBS [3]}

In order to improve the problem that will cause the TXOP to be wasted in the HCCA mechanism, the W-CBS in [3] divides the calculation into two steps. $Q_{\min }$ and $Q_{\max }$ are obtained to calculate the quantity of MSDU needed to be transmitted within the MSI time. Finally, via the establishment of the CWF (Choice Weight factor) weight value, the W-CBS scheme calculates the quantity of MSDU. The relevant equations are shown as Eq. (5), (6) and (7).

$$
\begin{gathered}
Q_{\text {min }}=\left\lceil\frac{\rho_{i} \times \mathrm{MSI}_{i}}{L_{i}}\right\rceil \\
Q_{\text {max }}=\frac{\mathrm{PR}_{i} \times \mathrm{MSI}_{i}}{\mathrm{ML}_{i}} \\
Q_{i}=Q_{\text {min }}+\operatorname{CWF}\left(Q_{\text {max }}-Q_{\text {min }}\right)
\end{gathered}
$$

where $\mathrm{PR}_{i}$ represents the peak data rate and $\mathrm{ML}_{i}$ is the maximum MSDU size. The CWF weight value is between 0 and 1 . The transmission time of one MSDU is calculated after $Q_{i}$ is obtained, as Eq. (8) and (9):

$$
\begin{gathered}
t_{N}=t_{\mathrm{DATA}}+t_{\mathrm{SIFS}}+t_{\mathrm{ACK}}+t_{\mathrm{SIFS}} \\
t_{\mathrm{DATA}}=\frac{\text { PHY header }+ \text { MAC header }+L_{i}}{R_{i}}
\end{gathered}
$$

where the $t_{\mathrm{DATA}}$ represents the time to transmit the data, $t_{\mathrm{SIFS}}$ is the time to transmit SIFS $t_{\mathrm{SIFS}}$ and $t_{\mathrm{ACK}}$ is the time to transmit ACK. PHY header and MAC header are the headers of PHY and MAC respectively. By calculating $Q_{i}$ and $t_{N}$, the quantity of TXOP that the QAP should distribute for the QSTA is determined as Eq. (10):

$$
\mathrm{TXOP}_{i}=Q_{i} \times t_{N}
$$

As shown in Fig. 1, the A blocks represent the TXOP for the QSTA, which is distributed by the QAP. Then, the QSTA transmit data within this period of time. Therefore, when QSTA1 transmits data within the time distributed by the QAP, but there is still some TXOP left (as the C blocks). In Fig. 1, QSTA2 does not need to wait for the transmission.

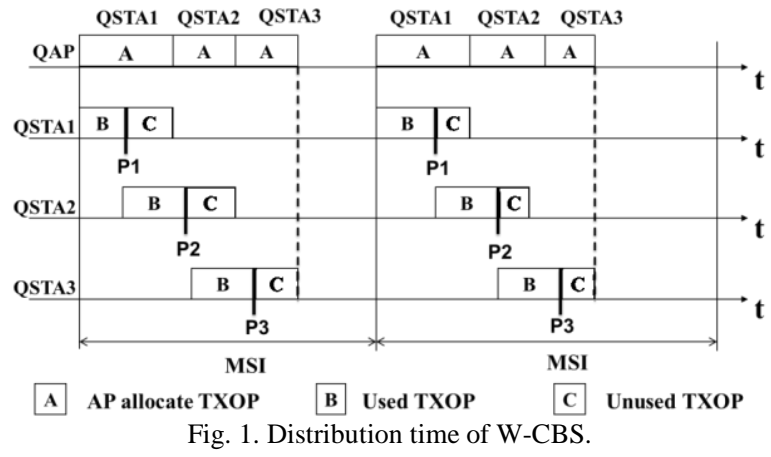

\section{X-TXOP (EXTRA-TXOP)}

The proposed X-TXOP mechanism can be divided into three parts. The first is the distribution of SI. The SI adopts the MSI to its schedule. The second part is the calculation of TXOP. Next, the X-TXOP mechanism calculates the TXOP via the QS. The transmission of QSTA will be discontinued by QAP due to the allocated TXOP. Therefore, the TXOP value is dynamically adjusted. The equations are shown as Eq. (11) and (12):

$$
\begin{gathered}
N_{i}=\left\lceil\frac{\mathrm{QS}_{i}+\mathrm{MSI}_{i} \times \rho_{i}}{L_{i}}\right\rceil \\
\operatorname{TXOP}_{i}=\frac{N_{i} \times L_{i}}{R_{i}}
\end{gathered}
$$

where the $\mathrm{QS}_{i}$ is the Queue Size value of $\mathrm{QSTA}_{i}$. After obtaining the TXOPs from the above equations, then the QAP distributes the $\mathrm{TXOP}_{i}$ to the $\mathrm{QSTA}_{i}$. As Eq. (13), the remaining TXOP of each QSTA will be summed and notified as $T_{\text {spare }}$.

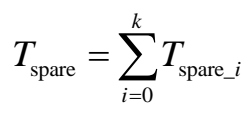

The third part of the X-TXOP mechanism is to distribute the sum of TXOP separately. There are two conditions for distributing $T_{\text {spare }}$. First, the TXOP obtained must be smaller

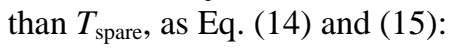

$$
\begin{gathered}
N_{i}=\left\lceil\frac{\mathrm{QS}_{i}}{L_{i}}\right\rceil \\
\mathrm{TXOP}_{i}=\frac{N_{i} \times L_{i}}{R_{i}}
\end{gathered}
$$

The second condition is to determine whether the packets in QSTA will be abandoned or not. In order to achieve this goal, the X-TXOP mechanism adopts the $\operatorname{Bit}(7)$ in QoS Control field to determine the QoS data frame. The process is shown as Eq. (16) and illustrated as Fig. 2.

$$
\begin{gathered}
\text { If } t_{\text {survival }}-\mathrm{MSI}_{i}<0 \\
\operatorname{Bit}(7)=1
\end{gathered}
$$


If there are more than one conditions established, the X-TXOP mechanism will automatically adopt the biggest TXOP first. Whenever the $T_{\text {spare }}$ is distributed to the QSTA, the X-TXOP mechanism must calculate the TXOP value of the QSTA again, as shown as Eq. (17) and (18):

$$
\begin{gathered}
N_{i}=\left\lceil\frac{\mathrm{QS}_{i}+\left(t_{\text {next_poll_i } i}-t_{\text {extra_poll_i } i}\right) \times \rho_{i}}{L_{i}}\right\rceil \\
\operatorname{TXOP}_{i}=\frac{N_{i} \times L_{i}}{R_{i}}
\end{gathered}
$$

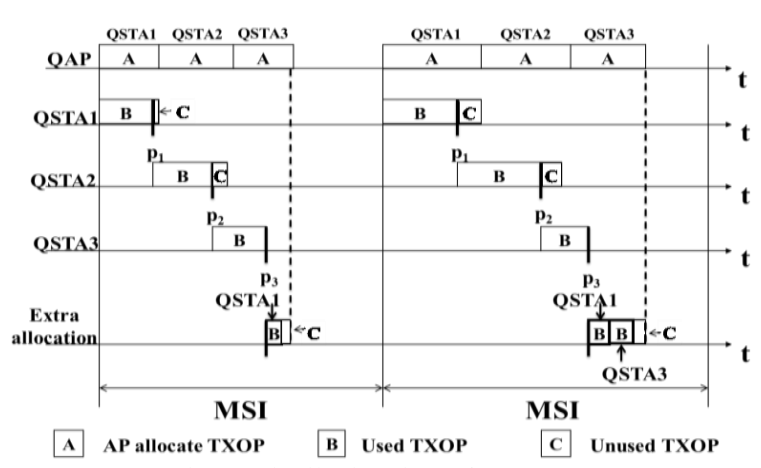

Fig. 2. Distribution time of X-TXOP.

Fig. 2 shows the distribution result of the X-TXOP mechanism. As shown in Fig. 2, the remaining TXOP will be allocated to QSTA to improve the utilization of the bandwidth.

\section{Simulation Results}

In simulation tests, we consider a LAN of one QAP and 1 10 QSTAs. There are two traffic flows of each QSTA in the simulations, including a Constant Bit Rate flow and a Variable Bit Rate flow. The 802.11 WLAN system parameters and TSPEC used in the simulations are shown in the Tables I and II.

TABLE I: PARAMETERS OF PHY/MAC
\begin{tabular}{|l|l|}
\hline Parameters & Value \\
\hline SIFS $(\mu \mathrm{s})$ & 10 \\
\hline SIFS $(\mu \mathrm{s})$ & 30 \\
\hline ASlotTime $(\mu \mathrm{s})$ & 20 \\
\hline Ack $($ Bytes $)$ & 16 \\
\hline CF-Poll $($ Bytes $)$ & 32 \\
\hline MAC header $($ Bytes $)$ & 36 \\
\hline PLCP header $($ Bytes $)$ & 6 \\
\hline PLCP preamble $($ Bytes $)$ & 8 \\
\hline Data rate $(\mathrm{Mb} / \mathrm{s})$ & 11 \\
\hline
\end{tabular}

\section{TABLE II: PARAMETERS OF CBR/VBR TSPEC}

\begin{tabular}{|l|l|l|}
\hline TSPEC & CBR traffic & VBR traffic \\
\hline Mean Data Rate (Kbps) & 83 & 128 \\
\hline Peak Data Rate (Kbps) & 83 & 1700 \\
\hline Nominal MSDU Size (bytes) & 208 & 1300 \\
\hline Minimum PHY Rate (Mb/s) & 11 & 11 \\
\hline Maximum Service Interval (ms) & 30 & 40 \\
\hline Delay Bound (ms) & 60 & 40 \\
\hline
\end{tabular}

\section{A. Analysis of Throughput}

In this experiment, we evaluate the performance improvement of the proposed X-TXOP mechanism within different network loads. The number of QSTA is set as 1 to 10 . Fig. 3 shows the system throughput for all related schemes. The figure reports the throughput increases when the number of QSTA increases. As mentioned in Section II, the IEEE 802.11e standard calculates the TXOP based on the speed of regular data transmission. Moreover, the W-CBS scheme provides higher throughput than IEEE 802.11e because of adopting the CWF weight value in the TXOP calculation. Finally, the proposed X-TXOP mechanism results in the highest throughput due to considering the queue size and remaining TXOP in determining of TXOP value.

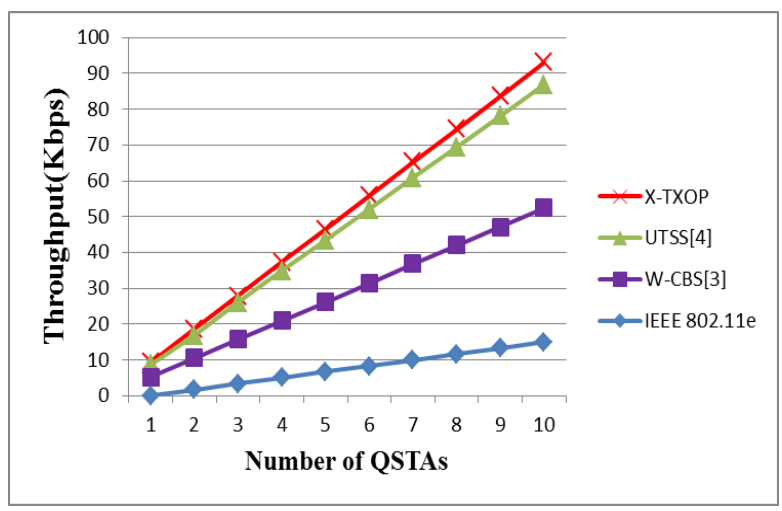

Fig. 3. Analysis of throughput.

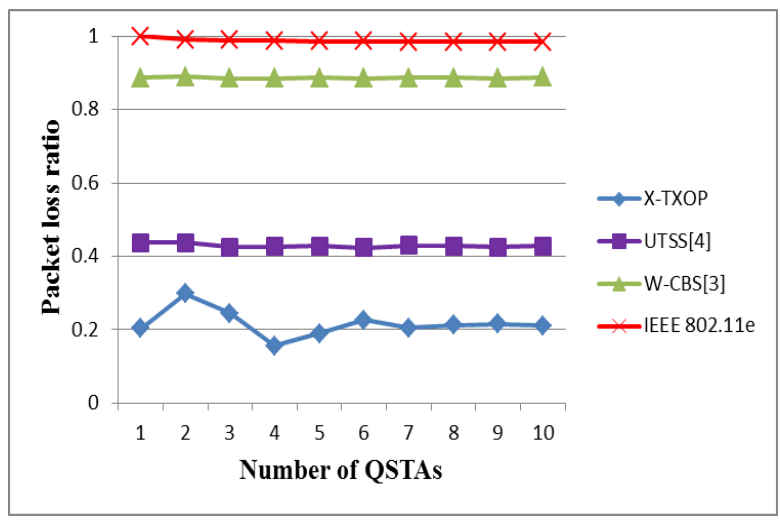

Fig. 4. Analysis of packet loss ratio.

\section{B. Analysis of Packet Loss Ratio}

From Fig. 4, we learn that the IEEE 802.11e standard is not suitable for VBR flow, because the standard adopts the average speed in calculating the TXOP. The W-CBS increases the period of TXOP, so the packet loss ratio is lower than IEEE 802.11e. The proposed X-TXOP mechanism provides lowest packet loss ratio of all because the X-TXOP utilizes the remaining X-TXOP to give more transmission chance to the QSTA which is in heavy load.

\section{CONCLUSION}

In this paper, we proposed X-TXOP mechanism to improve the system throughput and packet loss ratio. The X-TXOP utilizes the remaining TXOP of each QSTA and gives more transmission chance to the QSTA which is in heavy load. The numerical results show that the proposed X-TXOP mechanism improves system performance than IEEE $802.11 \mathrm{e}$ and $\mathrm{W}-\mathrm{CBS}$. 


\section{ACKNOWLEDGEMENTS}

We would like to thank the National Science Council (NSC) in Taiwan. This research was supported in part by the NSC under the Grant No.MOST104-2221-E-151-005.

\section{REFERENCES}

[1] IEEE Standard for Information Technology - Telecommunications and Information Exchange between Systems Local and Metropolitan Area Networks - Specific Requirements Part 11: Wireless LAN Medium Access Control (MAC) and Physical Layer (PHY) Specifications, IEEE Standard Association.

[2] IEEE Standard for Information Technology - Telecommunications and Information Exchange between Systems Local and Metropolitan Area Networks - Specific Requirements Part 11: Wireless LAN Medium Access Control (MAC) and Physical Layer (PHY) Specifications Amendment 8: Medium Access Control (MAC) Quality of Service Enhancements, IEEE Standard Association.

[3] G. Cecchetti, A. L. Ruscelli, and F. Checconi, "W-CBS: A scheduling algorithm for supporting QoS in IEEE 802.11e," in Proc. the Fourth International Conference on Heterogeneous Networking for Quality, Reliability, Security and Robustness \& Workshops, 2007.

[4] A. L. Ruscelli, G. Cecchetti, G. Lipari, and A. Mastropaolo, "A greedy reclaiming scheduler for IEEE 802.11e HCCA real-time networks," in Proc. the 14th ACM International Conference on Modeling, Analysis and Simulation of Wireless and Mobile Systems, 2011, pp. 223-230.

[5] A. Grilo, M. Macedo, and M. Nunes, "A scheduling algorithm for QoS support in IEEE 802.11E networks," IEEE Wireless Communications, 2003.

[6] D. Skyrianoglou, N. Passas, and A. K. Salkintzis. "ARROW: An efficient traffic scheduling algorithm for IEEE 802.11e HCCA," IEEE Transactions on Wireless Communications, vol. 5, no. 12, pp. 3558-3567, 2006.

[7] Q. Zhao, "An equal-spacing-based design for QoS guarantee in IEEE 802.11e HCCA wireless networks," Mobile Computing IEEE Transactions, vol. 7, no. 12, pp. 1474-1490, 2008.

[8] A. A. Bourawy, N. A. A. Ali, and H. S. Hassanein, "A selectivity function scheduler for IEEE 802.11e," in Proc. of IEEE Symposium of Computers and Communications, 2009, pp. 950-959.

[9] A. L. Ruscelli, G. Cecchetti, A. Alifano, and G. Lipari, "Enhancement of QoS support of HCCA schedulers using EDCA function in IEEE 802.11e networks," Ad Hoc Networks, vol. 10, no. 2, pp. 147-161, 2010.

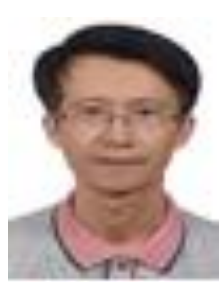

Ming-Hua Cheng received his M.I.M. degree in 2006 from the Department of Management Information Systems, National Pingtung University of Science and Technology and the B.M.S. degree in 1996 from the Department of Industrial and Information Management, National Cheng Kung University, Tainan. His current research interests include wireless network, LTE 5G, software defined network (SDN), and internet of thing (IOT).

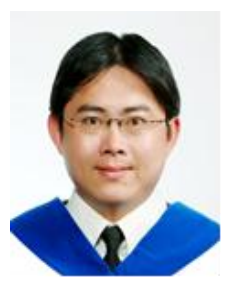

Cheng-Han Lin received the $\mathrm{PhD}$ degree from the Department of Electrical Engineering, National Cheng Kung University in 2010 and the MS and BS degrees from the Electrical Engineering Department of National Chung Cheng University in 2002 and 2004 respectively. He is currently an assistant professor of the Program of Information Technology, Fooyin University, Taiwan. He was a post doctor in National Kaohsiung University of Applied Sciences, from 2010 to 2012, and National Cheng Kung University, from 2012 to 2015. His current research interests include wireless network, multimedia communications, and SDN network.

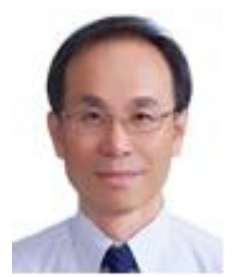

Wen-Shyang Hwang received his B.S., M.S., and $\mathrm{Ph} . \mathrm{D}$. degrees in the Electrical Engineering Department of National Cheng Kung University, Taiwan, in 1984, 1990, and 1996, respectively. He is currently a professor with the Electrical Engineering Department of National Kaohsiung University of Applied Sciences (KUAS), he was the chief of the Computer Science and Information Engineering Department of KUAS from 2006 to 2009, and was the director of the Computer and Network Center of KUAS from 2010 to 2012, Taiwan. Moreover, professor Hwang was the secretary general (from 2009 to 2013) and since 2013, he has been a council member of Chinese Institute of Electrical Engineering at Kaohsiung; he also is a council member of IEEE at Tainan Section (from 2014 to 2015). His current research interests are in the fields of multimedia wireless communication, wireless mesh networks, storage area networks, WDM metro-ring networks, performance evaluation, software design for embedded systems, internet QoS, and internet applications.

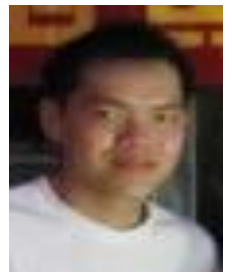

Feng-Cheng Yu received M.S. degrees in the Electrical Engineering Department of National Kaohsiung University of Applied Sciences, Tainan, in 2013. His current research interests include wireless network and multimedia communications. 